Pacific

Journal of

Mathematics

A GENERALIZED BURAU REPRESENTATION FOR STRING LINKS

Daniel S. Silver and Susan G. Williams

Volume $197 \quad$ No. 1

January 2001 


\title{
A GENERALIZED BURAU REPRESENTATION FOR STRING LINKS
}

\author{
Daniel S. Silver and Susan G. Williams
}

\begin{abstract}
A 2-variable matrix $\mathcal{B} \in \mathrm{GL}_{n}\left(\mathrm{Z}\left[u^{ \pm 1}, v^{ \pm 1}\right]\right)$ is defined for any $n$-string link, generalizing the Burau matrix of an $n$ braid. The specialization $u=1, v=t^{-1}$ recovers the generalized Burau matrix recently defined by X. S. Lin, F. Tian and $\mathrm{Z}$. Wang using probabilistic methods. The specialization $u=t^{-1}, v=1$ results in a matrix with a natural algebraic interpretation, and one that yields homological information about the complement of the closed string link.
\end{abstract}

\section{Introduction.}

An $n$-string link, for $n$ a positive integer, is a collection of $n$ embedded strings in $\mathbf{R}^{2} \times[0,1]$ such that the $i$ th string joins a point $P_{i} \times 1$ in the plane $\mathbf{R}^{2} \times 1$ to a point $P_{\pi i} \times 0$ directly below in the parallel plane $\mathbf{R}^{2} \times 0$. We visualize string links by diagrams such as in Figure 1.

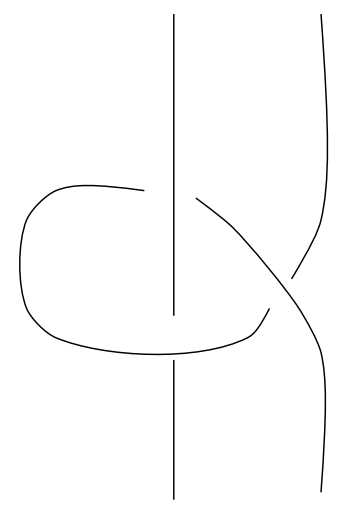

Figure 1. A 2-String Link.

Two $n$-string links are regarded as the same if a diagram for one can be obtained from a diagram for the other by a finite sequence of Reidemeister moves (Figure 5). The set of all $n$-string links has the structure of a monoid $S_{n}$ under concatenation. When each string meets every plane $\mathbf{R}^{2} \times t, 0 \leq$ 
$t \leq 1$, transversely in a single point, the string link is also called an $n$ braid. The set of all $n$-braids is a group $B_{n}$ with generators $\sigma_{1}, \ldots, \sigma_{n-1}$ and relations as in Figure 2.

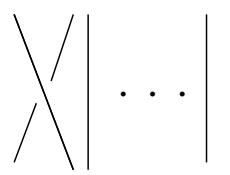

$\sigma_{1}$

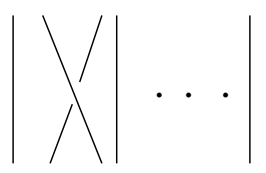

$\sigma_{2}$

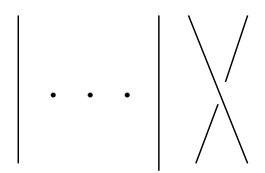

$\sigma_{\mathrm{n}-1}$

$$
\begin{aligned}
& \sigma_{i} \sigma_{i+1} \sigma_{i}=\sigma_{i+1} \sigma_{i} \sigma_{i+1} \quad \text { for } \quad 1 \leq i \leq n-2 \\
& \sigma_{i} \sigma_{j}=\sigma_{j} \sigma_{i} \quad \text { for } \quad|i-j| \geq 2
\end{aligned}
$$

Figure 2. Generators and Relations for $B_{n}$.

The Burau representation $B: B_{n} \rightarrow G L\left(n, \mathbf{Z}\left[t^{ \pm 1}\right]\right)$ maps $\sigma_{i}$ to the block diagonal matrix

$$
B_{\sigma_{i}}(t)=\left(\begin{array}{cccc}
I_{i-1} & & & \\
& 1-t & t & \\
& 1 & 0 & \\
& & & I_{n-i-1}
\end{array}\right),
$$

where $I_{k}$ denotes the $k \times k$ identity submatrix. The entries of $B_{\alpha}(t)$ are Laurent polynomials $b_{i, j}(t)$. (See [Bi], for example.) In [LiTiWa] the authors consider V. Jones' probabilistic interpretation of $B_{\sigma}(t)[\mathbf{J} \mathbf{o}]$ in order to extend the Burau representation to a representation $\bar{B}$ of the monoid $S_{n}$.

In a sense there are two Burau representations. It is a consequence of the defining relations of $B_{n}$ that the map sending each $\sigma_{i}$ to the transpose $B_{\sigma_{i}}(t)^{T}$ determines another representation $B^{\prime}: B_{n} \rightarrow G L\left(n, \mathbf{Z}\left[t^{ \pm 1}\right]\right)$. The two Burau representations are related by the following diagram.

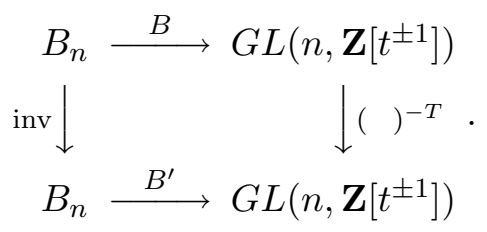

Here inv denotes the automorphism of $B_{n}$ that maps each generator $\sigma_{i}$ to its inverse, while ()$^{-T}$ is the automorphism of $G L\left(n, \mathbf{Z}\left[t^{ \pm 1}\right]\right)$ that sends each matrix to its inverse transpose.

The representations $B$ and $B^{\prime}$ have subtle differences, the latter being more suitable for colorings and computation of Alexander invariants. In [Ka] Kauffman extracts $B^{\prime}$ from the Alexander crystal.

We will show that the representations $B$ and $B^{\prime}$ are in fact two specializations of a single representation $\mathcal{B}: B_{n} \rightarrow G L\left(n, \mathbf{Z}\left[u^{ \pm 1}, v^{ \pm 1}\right]\right)$, described 
in Section 2. Setting $u=1, v=t^{-1}$ yields $B$, while setting $u=t^{-1}, v=1$ produces $B^{\prime}$. We will show that $\mathcal{B}$ extends to a representation $\overline{\mathcal{B}}$ of $S_{n}$. Then the specialization $u=1, v=t^{-1}$ results in the extension $\bar{B}$ of $[\mathbf{L i T i W a}$, while setting $u=t^{-1}, v=1$ produces an extension of $B^{\prime}$ that can be used to gain homological information about the complement of the closed string link. C. Livingston informs us that he, P. Kirk and Z. Wang discovered a similar 2-variable Burau representation while writing [KiLiWa].

In the last section we associate a sequence of "algebraic entropies" $\gamma_{\alpha, r}$ to any string link $\alpha$, for $r \in \mathbf{N}$. In the case of a braid $\alpha$, these quantites are lower bounds for the usual braid entropy. Let $\alpha$ be a string link with strings coherently oriented, and let $l_{m}$ denote the oriented knot or link obtained as the closure of the product $\alpha^{m}, m \geq 1$. We prove that $\gamma_{\alpha, r}$ is equal to the exponential growth rate of $\left|T H_{1}\left(M_{r}\left(l_{m}\right) ; \mathbf{Z}\right)\right|$, where $M_{r}\left(l_{m}\right)$ is the $r$-fold cyclic cover of $S^{3}$ branched over $l_{m}$, and $T(\cdot)$ denotes the torsion subgroup. This result extends Theorem 3.2 of [ $\mathbf{S i W i}$ ] .

We are grateful to J. Scott Carter and the referee for their comments and suggestions.

\section{The $\Lambda$-group of a string link.}

Let $\Lambda$ denote the free abelian group of rank 2 generated by $u$ and $v$. A $\Lambda$ group is a group $K$ with a right action $K \times \Lambda \rightarrow K$, denoted by $(g, w) \mapsto g^{w}$. We define $\Lambda$-subgroup, $\Lambda$-homomorphism, etc. in the obvious way (see [Rob]).

Let $D$ be a diagram for an $n$-string link $\alpha$. We orient $D$ so that each string is directed from top to bottom. Place vertices on $D$ so that in a neighborhood of any crossing two vertices "enter" and two vertices "exit." If $D$ has $N$ crossings, we use exactly $n+2 N$ vertices. We label the vertices $a, b, c, \ldots$, as in Figure 3 .

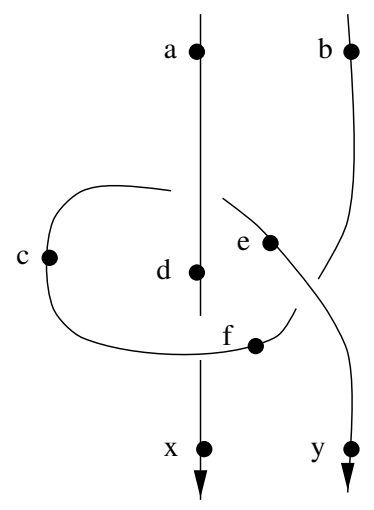

Figure 3. Labeled String Link. 
To the diagram $D$ we associate a $\Lambda$-group $K$, described by a presentation. Each vertex label of $D$ determines a $\Lambda$-generator; that is, a set of generators indexed by $\Lambda$. Thus the letter $a$ denotes a set $\left\{a_{j, k}\right\}$ of generators $a_{j, k}=$ $a^{u^{j} v^{k}}$, where $j, k$ range over $\mathbf{Z}$. Each crossing of $D$ determines a pair of $\Lambda$ relations, described in Figure 4. A $\Lambda$-relation is a set of relations indexed by $\Lambda$. For example, $a^{u} b=c^{u} d$ denotes the set of relations of the form $a_{j+1, k} b_{j, k}=c_{j+1, k} d_{j, k}$, where $j, k$ range over the integers. Likewise $b^{v}=c$ denotes the set of relations of the form $b_{j, k+1}=c_{j, k}$.
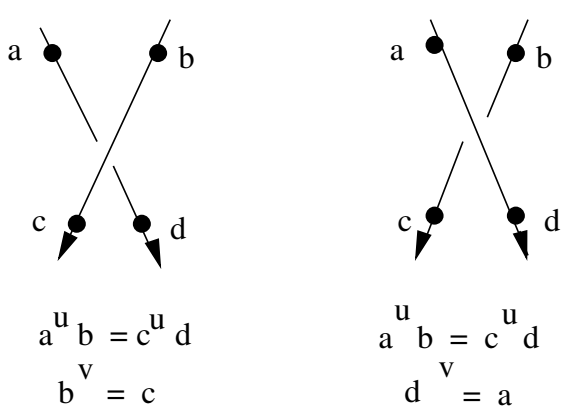

Figure 4. $\Lambda$-Relations for $K$.

We also distinguish two $n$-tuples of $\Lambda$-generators, the vector $\mathcal{S}$ corresponding to input vertices (those on the top of the diagram) and the vector $\mathcal{T}$ corresponding to output vertices (those on the bottom).

The abelianized group $K_{a b}$ is a finitely generated $\Lambda$-module. The two relations determined by a crossing as in Figure 4 are equivalent to:

$$
\begin{aligned}
u a+(1-u v) b=d, v b=c, & \text { if } \epsilon=+1 \\
\left(1-u^{-1} v^{-1}\right) a+u^{-1} b=c, v^{-1} a=d, & \text { if } \epsilon=-1
\end{aligned}
$$

where $\epsilon= \pm 1$ is the sign of the crossing.

Relations (2.1) can be written in matrix form $(a, b) M^{\epsilon}=(c, d)$, where

$$
M=\left(\begin{array}{cc}
0 & u \\
v & 1-u v
\end{array}\right) .
$$

In [CaSa] J. Scott Carter and M. Saito observed that a family of invertible $2 \times 2$ matrices $X$ including $M$ satisfies the equation

$$
(X \oplus 1)(1 \oplus X)(X \oplus 1)=(1 \oplus X)(X \oplus 1)(1 \oplus X) .
$$

They call $X$ a "twisted Burau matrix."

Example 2.1. For the diagram in Figure 3,

$$
\begin{array}{r}
K=\langle a, b, c, d, e, f, x, y| \\
\left.c^{u} a=d^{u} e, a^{v}=d, e^{u} b=f^{u} y, y^{v}=e, d^{u} f=c^{u} x, f^{v}=c\right\rangle .
\end{array}
$$


Here $\mathcal{S}=(a, b)$ and $\mathcal{T}=(x, y)$. Using the second, fourth and sixth relations we can eliminate the generators $d, e$ and $c$, thereby obtaining the simpler presentation

$$
K=\left\langle a, b, f, x, y \mid f^{u v} a=a^{u v} y^{v}, y^{u v} b=f^{u} y, a^{u v} f=f^{u v} x\right\rangle .
$$

We can use the first relation to eliminate $f$, getting

$$
K=\left\langle a, b, x, y \mid y^{u v^{2}} b^{v}=a^{u v} y^{v} a^{-1} y^{v}, a^{u v} y^{v} a^{-1}=y^{u v^{2}}\left(a^{-1}\right)^{u v} x^{u v}\right\rangle .
$$

Proposition 2.2. If $(K ; \mathcal{S}, \mathcal{T})$ and $\left(K^{\prime} ; \mathcal{S}^{\prime}, \mathcal{T}^{\prime}\right)$ are associated to diagrams of the same $n$-string link, then there exists a $\Lambda$-isomorphism $f: K \rightarrow K^{\prime}$ taking $(\mathcal{S}, \mathcal{T})$ to $\left(\mathcal{S}^{\prime}, \mathcal{T}^{\prime}\right)$.

Proof. Consider the three basic Reidemeister moves (Figure 1). It suffices to prove that in each case the expressions for the two $\Lambda$-generators at the bottom in terms of the two at the top, expressions that result from the crossing relations in the diagram, are unchanged after the move is applied; the remaining generators in each case can be eliminated. The verification is trivial for the first and second Reidemeister moves, regardless of how the strings are oriented.

Invariance under the second Reidemeister move with strings oppositely oriented is termed "cross-channel unitarity" in $[\mathbf{K a}]$. It is shown there that if one has cross-channel unitarity for any quantity, then invariance of that quantity under the third Reidemeister move with strings oriented in the same direction implies invariance under that move with any string orientations. The verification for coherently oriented strings is straightforward.

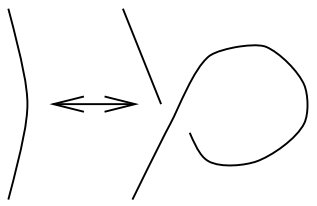

I
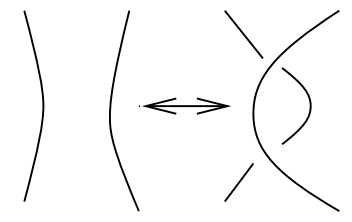

II

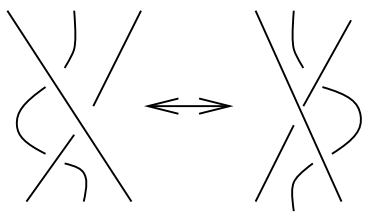

III

Figure 5. Reidemeister Moves.

In view of Proposition 2.2 the triple $(K ; \mathcal{S}, \mathcal{T})$ is an invariant of the $n$ string link $\alpha$. Whenever $(K ; \mathcal{S}, \mathcal{T})$ is a triple associated to an $n$-braid, $K$ is a free $\Lambda$-group. Moreover $\mathcal{S}$ and $\mathcal{T}$ are ordered bases for $K$. The classical Burau representation compares $\mathcal{S}$ and $\mathcal{T}$ in a certain quotient of $K$, as we will see.

Example 2.3. Consider the 3-braid $\sigma_{1}^{-1} \sigma_{2}$. A labeled diagram for this braid appears in Figure 6 . Here $\mathcal{S}=(a, b, c)$ and $\mathcal{T}=(x, y, z)$. The associated $\Lambda$-group $K$ has presentation

$$
K=\left\langle a, b, c, d, x, y, z \mid a^{u} b=x^{u} d, b^{v}=x, d^{u} c=y^{u} z, z^{v}=d\right\rangle .
$$




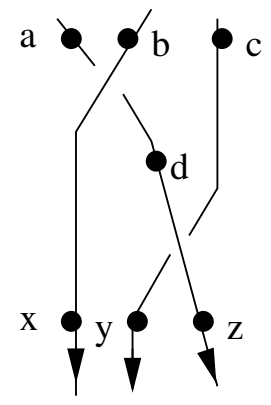

Figure 6. Labeled 3-braid $\sigma_{1}^{-1} \sigma_{2}$.

Using the fourth relation we eliminate the generator $d$, obtaining

$$
K=\left\langle a, b, c, x, y, z \mid a^{u} b=x^{u} z^{v}, b^{v}=x, z^{u v} c=y^{u} z\right\rangle .
$$

It is clear from the last presentation that $K$ is a free $\Lambda$-group of rank 3 generated by $a, b, c$ and also by $x, y, z$. We will now consider the abelianized group $K_{a b}$, regarded as a $\Lambda$-module. Let $\mathcal{B}$ denote the change of basis matrix such that $(a, b, c) \mathcal{B}=(x, y, z)$. Then

$$
\mathcal{B}=\left(\begin{array}{ccc}
0 & u^{2}-u v^{-1} & u v^{-1} \\
v & 2 u-u^{2} v-v^{-1} & v^{-1}-u \\
0 & 1 & 0
\end{array}\right) .
$$

Setting $u=1$ and $v=t^{-1}$ recovers the Burau matrix $B\left(\sigma_{1}^{-1} \sigma_{2}\right)$,

$$
B\left(\sigma_{1}^{-1} \sigma_{2}\right)=\left(\begin{array}{ccc}
0 & 1-t & t \\
t^{-1} & 2-t^{-1}-t & t-1 \\
0 & 1 & 0
\end{array}\right) .
$$

It is not difficult to see that for any $n$-braid generator $\sigma_{i}$ the change of basis matrix, defined as in Example 2.3, yields the Burau matrix $B\left(\sigma_{i}\right)$ when the substitution $u=1, v=t^{-1}$ is made. (Hence the result holds for any $n$-braid $\alpha$.) It is also clear that the substitution $u=t^{-1}, v=1$ results in the transpose $B\left(\sigma_{i}\right)^{T}$.

Assume that the triple $(K ; \mathcal{S}, \mathcal{T})$ is associated to an $n$-string link $\alpha$. We will regard $K_{a b}$ as a $\Lambda$-module. Since $\Lambda$ is a commutative domain (see, for example, Theorem 2.1.3 of [Ja], Volume 1), it embeds in the field $\mathbf{Q}(u, v)$ of rational expressions in $u, v$. The generators of $K$ will be regarded as elements of the vector space $K_{a b} \otimes_{\Lambda} \mathbf{Q}(u, v)$ via the natural map $w \mapsto w \otimes 1, w \in K_{a b}$; in particular, $\mathcal{S}$ and $\mathcal{T}$ are regarded as $n$-tuples of elements of $K_{a b} \otimes_{\Lambda} \mathbf{Q}(u, v)$.

Proposition 2.4. Assume that the triple $(K ; \mathcal{S}, \mathcal{T})$ is associated to an $n$ string link $\alpha$. The vector space $K_{a b} \otimes_{\Lambda} \mathbf{Q}(u, v)$ has dimension $n$. Furthermore, $\mathcal{S}$ and $\mathcal{T}$ are ordered bases. 
Proof. Consider a presentation for $K_{a b}$ obtained from a diagram for $\alpha$. Order the generators so that the coordinates $a, b, \ldots$ of $\mathcal{S}$ are first, and the coordinates $x, y, \ldots$ are second. (By applying Reidemeister moves to the diagram we can assume that the coordinates of $\mathcal{S}$ are disjoint from those of $\mathcal{T}$.) The relation matrix has $2 N$ columns, corresponding to the $N$ crossings of $D$; it has $2 N+n$ rows, corresponding to the generators of $K_{a b}$. Let $d(u, v)$ be the determinant of the $2 N \times 2 N$ submatrix obtained by deleting the first $n$ rows.

If we set $u=v=1$, then it is clear from the resulting form of the relations that every generator can be uniquely expressed in terms of coordinates $a, b, \ldots$ of $\mathcal{S}$. Consequently, $d(1,1)$ is nonzero. It follows that $d(u, v)$ must be nonzero, and hence the conclusions of Proposition 2.4 hold.

Example 2.5. We return to the string link of Example 2.1. Using the presentation (2.3) we see that the $\Lambda$-module $K_{a b}$ has presentation

$$
\begin{aligned}
K_{a b}=\langle a, b, x, y|\left(u v^{2}-2 v\right) y=(u v-1) a-v b & \\
& \left.u v x+\left(u v^{2}-v\right) y=(2 u v-1) a\right\rangle .
\end{aligned}
$$

Consider the vector space $K_{a b} \otimes_{\Lambda} \mathbf{Q}(u, v)$, and let $\mathcal{B}$ be the change of basis matrix such that $\mathcal{S B}=\mathcal{T}$, where $\mathcal{S}=(a, b)$ and $\mathcal{T}=(x, y)$. A routine calculation reveals that

$$
\mathcal{B}=\frac{1}{2-u v}\left(\begin{array}{cc}
-1+3 u v-u^{2} v^{2} & v^{-1}-u \\
u^{-1}-v & 1
\end{array}\right) .
$$

Motivated by Example 2.5, we state our main definition.

Definition 2.6. Let $\alpha$ be an $n$-string link with associated triple $(K ; \mathcal{S}, \mathcal{T})$. The two variable Burau matrix of $\alpha$ is the change of basis matrix $\mathcal{B}=$ $\mathcal{B}(u, v)$ such that $\mathcal{S B}=\mathcal{T}$ in the vector space $K_{a b} \otimes_{\Lambda} \mathbf{Q}(u, v)$.

\section{String links and paths.}

If we make the substitution $u=1, v=t$ in Example 2.5 the resulting matrix $\mathcal{B}(1, t)$ is essentially that defined in $[\mathbf{L i T i W a}]$. The difference arises from string orientation. (We have chosen to flow from top to bottom.) We will show that this is generally the case by a combinatorial description of $\mathcal{B}$ generalizing the approach of $[\mathbf{L i T i W a}]$.

Let $D$ be a diagram of an $n$-string link with strands oriented from top to bottom and with vertex labels as in Section 2. If $V_{i}$ and $V_{j}$ are vertices, we will write $V_{i} \rightarrow V_{j}$ if (i) $V_{i}$ and $V_{j}$ are on the same arc, with orientation from $V_{i}$ to $V_{j}$; (ii) $V_{i}$ and $V_{j}$ are on arcs separated by an overcrossing, with orientation from $V_{i}$ to $V_{j}$; or (iii) $V_{j}$ is reached by moving along the arc containing $V_{i}$ in the preferred direction and then jumping down after the crossing to the arc below. These transitions are illustrated in Figure 7. We assign weights $w\left(V_{i}, V_{j}\right) \in \Lambda$ as indicated. 


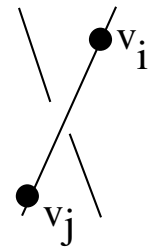

$\mathrm{V}$

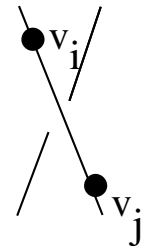

$\mathrm{v}^{-1}$

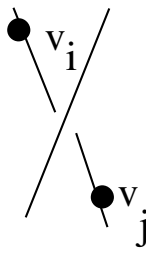

$\mathrm{u}$

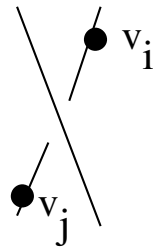

$u^{-1}$

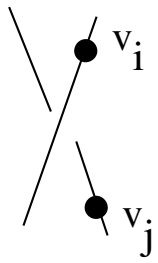

$1-\mathrm{uv}$

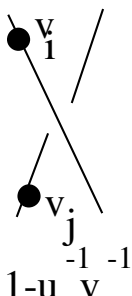

$1-\mathrm{u}$ v

(i)

(ii)

(iii)

Figure 7. Transitions $V_{i} \rightarrow V_{j}$ and Weights.

If $V$ and $V^{\prime}$ are vertices of $D$, then a path $P$ from $V$ to $V^{\prime}$ is a finite sequence $V=V_{0} \rightarrow V_{1} \rightarrow \cdots \rightarrow V_{k}=V^{\prime}$. We denote the product $w\left(V_{0}, V_{1}\right) w\left(V_{1}, V_{2}\right) \cdots w\left(V_{k-1}, V_{k}\right)$ by $w(P)$.

The following result is a generalization of the observation preceding Proposition 2.1 in [LiTiWa].

Lemma 3.1. For any vertices $V, V^{\prime}$ of $D$, the sum

$$
\sum_{P} w(P)
$$

over all paths from $V$ to $V^{\prime}$ converges to a formal power series of the form

$$
\sum_{m, n=1}^{\infty} A_{m, n}(1-u)^{m}(1-v)^{n}
$$

Proof. We adopt some terminology of $[\mathbf{L i T i W a}]$. A loop is a path that begins and ends at the same vertex; it is simple if no proper segment is a loop. The multiplicity of a path $P$ is the number of simple loops that it contains.

The weight of any simple loop contains a factor of $1-u v$ or $1-u^{-1} v^{-1}$. Note that

$$
\begin{aligned}
1-u v & =(1-u)+(1-v)-(1-u)(1-v) \\
1-u^{-1} v^{-1} & =\left(1-u^{-1}\right)+\left(1-v^{-1}\right)-\left(1-u^{-1}\right)\left(1-v^{-1}\right) .
\end{aligned}
$$

The right-hand side of the second expression can be written as

$$
-\sum_{m=1}^{\infty}(1-u)^{m}-\sum_{n=1}^{\infty}(1-v)^{n}-\left[\sum_{m=1}^{\infty}(1-u)^{m}\right]\left[\sum_{n=1}^{\infty}(1-v)^{n}\right]
$$

which is easily seen to be equal to

$$
-\sum_{m=1}^{\infty}(1-u)^{m}-\sum_{n=1}^{\infty}(1-v)^{n}-\sum_{m, n=1}^{\infty}(1-u)^{m}(1-v)^{n} .
$$


Consequently, if $P$ is a path of multiplicity $k$, then the total degree in $1-$ $u, 1-v$ of each term of $w(P)$ is no less than $k$. Since there are only finitely many paths $P$ of multiplicity $k$, the power series (3.1) converges.

In order to accomodate the notational demands of this section, we relabel the generators of $K_{a b}$ by $s_{1}, \ldots, s_{n}, t_{1}, \ldots, t_{n}, \ldots t_{2 N}$ with $\mathcal{S}=\left(s_{1}, \ldots, s_{n}\right)$ and $\mathcal{T}=\left(t_{1}, \ldots, t_{n}\right)$. (See parenthetical remark in proof of Proposition 2.4.) As before $N$ is the number of crossings of $D$. Fix indices $i, j$ with $1 \leq i \leq n$ and $1 \leq j \leq 2 N$, and consider the set of all paths $P$ from the vertex labeled $s_{i}$ to that labeled $t_{j}$. Let $W_{i, j}$ be the sum of the weights $w(P)$ of all such paths.

A priori, $W_{i, j}$ is an element of the ring $\mathbf{Z}[[u-1, v-1]]$ of formal power series in $u-1, v-1$. This ring is contained in the field $\mathbf{Q}((u-1, v-1))$ of formal Laurent series over $\mathbf{Q}$, a field that also contains the rational expressions $\mathbf{Q}(u, v)(\operatorname{see}[\mathbf{J a}])$.

Proposition 3.2. For each $1 \leq i, j \leq n$, the quantity $W_{i, j}$ is an invariant of the string link, equal to the $i, j$ entry of the generalized Burau matrix $\mathcal{B}$.

Proof. In the proof of Proposition 2.4 we regarded the generators of $K_{a b}$ as elements of $K_{a b} \otimes_{\Lambda} \mathbf{Q}(u, v)$. Now we regard them as elements of the larger vector space $K_{a b} \otimes \mathbf{Q}((u, v))$. For each $j=1, \ldots, 2 N$ set

$$
\bar{t}_{j}=\sum_{i=1}^{n} W_{i, j} s_{i} .
$$

We see easily that $s_{1}, \ldots, s_{n}, \bar{t}_{1}, \ldots, \bar{t}_{2 N}$ satisfy the same relations (2.1) as $s_{1}, \ldots, s_{n}, t_{1}, \ldots, t_{2 N}$. Since $s_{1}, \ldots, s_{n}$ is a basis for $K_{a b} \otimes \mathbf{Q}((u, v))$, we must have $\bar{t}_{j}=t_{j}$, for all $j=1, \ldots, 2 N$. In particular, this is true for $1 \leq j \leq n$.

In the case of an $n$-string link with diagram $D$ having only positive crossings, the $i, j$ entry of $\mathcal{B}$ acquires the probabilistic interpretation of [LiTiWa] if one sets $u=1$ and $v=1 / p$, where $0 \leq p \leq 1$. Regarding any weight $w\left(V_{i}, V_{j}\right)$ as the probability that a particle at vertex $V_{i}$ goes immediately to $V_{j}$, the entry $W_{i, j}$, for $1 \leq i, j \leq n$, is then the probability that a particle starting on the top of the $i$ th string will exit at the bottom of the $j$ th string.

\section{Algebraic entropy of a string link.}

Let $(K ; \mathcal{S}, \mathcal{T})$ be a triple associated to an $n$-string link $\alpha$, as above. Let $s$ be a new variable, and consider the $\Lambda[s]$-group $L$ obtained from $K$ by introducing $n$ relations $a^{s}=x, b^{s}=y, \ldots$, corresponding to the coordinates of $\mathcal{S}$. It follows from the Reidemeister-Schreier method that when $s=u=1$ 
and $v=t^{-1}$, the group $L$ is isomorphic to the augmentation subgroup $\pi_{1}\left(S^{3}-l\right)$, where $l$ is the closure of $\alpha$ together with an unknotted distant component. (Details can be found in $[\mathbf{S i W i}]$.)

The abelianization $L_{a b}$ has a square presentation matrix. We will denote the determinant of the matrix by $\Delta(s, u, v)$. By the theory of finitely generated modules over a P.I.D. the polynomial $\Delta(s, u, v)$ is well defined up to multiplication by units in $\Lambda[s]$ (see $[\mathbf{J a}]$ ). When $\alpha$ is an $n$-braid and $u=1, v=t^{-1}$, the matrix $s I-B(\alpha)(t)$ presents $L_{\mathrm{ab}}$. Hence $\Delta\left(s, 1, t^{-1}\right)$ is equal to the characteristic polynomial of $B(\alpha)(t)$.

For any string link, the polynomial $\Delta(s, u, v)$ has the form $\Delta_{n}(u, v) s^{n}+$ $\cdots+\Delta_{1}(u, v) s+\Delta_{0}(u, v)$.

Definition 4.1 ([Ma] (see also $[\mathbf{S c}])$ ). The Mahler measure of a polynomial $f(s)=f_{n} s^{n}+\cdots+f_{1} s+f_{0}\left(f_{n} \neq 0\right)$ with complex coefficients is

$$
\mathcal{M}(f(s))=\left|f_{n}\right| \cdot \prod_{j=1}^{n} \max \left(\left|z_{j}\right|, 1\right),
$$

where $z_{1}, \ldots, z_{n}$ are the roots of $f(s)$.

Remark. The Mahler measure of $f(s)$ is equal to

$$
\exp \int_{\mathbf{T}^{1}} \log |f(s)| d s,
$$

where the integration is peformed over the unit circle $\mathbf{T}^{1}[\mathbf{M a}]$. It can be regarded as the geometric mean of $f$ over $\mathbf{T}^{1}$.

Definition 4.2. Let $\alpha$ be an $n$-string link with polynomial $\Delta(s, u, v)$, and let $r$ be a positive integer. The $r$ th algebraic entropy $\gamma_{\alpha, r}$ is

$$
\max _{0 \leq i, j<r} \log \mathcal{M}\left(\Delta\left(s, \zeta^{i}, \zeta^{j}\right)\right),
$$

where $\zeta$ is a primitive $r$ th root of unity.

Example 4.3. Consider $\alpha=\sigma_{1}^{-1} \sigma_{2}$ (Figure 6). The $\Lambda[s]$-module $L_{a b}$ has presentation

$$
\begin{aligned}
\langle a, b, c, d, x, y, z| a u+b=u x+d, v x=b, u d+c=u y+z & \\
& v d=z, x=s a, y=s b, z=s c\rangle
\end{aligned}
$$

(compare calculations found in $[\mathbf{K a}]$ ). Using the last four relations we can eliminate the generators $d, x, y, z$. We see

$$
\begin{aligned}
L_{a b}=\langle a, b, c|(s u-u) a-b+s v^{-1} c & =0, \\
s v a-b & \left.=0, s u b+\left(s-s u v^{-1}-1\right) c=0\right\rangle .
\end{aligned}
$$


A routine calculation shows that

$$
\Delta(s, u, v)=\frac{1}{v}(s-1)\left[u v s^{2}+\left(u^{2}-u v+v^{2}\right) s+u v\right],
$$

and from this it is easy to compute various algebraic entropies. For example, $\gamma_{\alpha, 2}=\log \frac{3+\sqrt{5}}{2}$.

Topological entropy $h(f)$ is defined for any continuous map $f$ of a compact space [AdKoMc]. It measures the dynamical complexity of the map. The concept has a natural extension for braids that is well known.

The $n$-braid group $B_{n}$ is isomorphic to the mapping class group $\operatorname{MCG}\left(D\right.$ rel $\left.P_{n}, \partial D\right)$, where $D$ is the 2 -disk and $P_{n}$ is constists of $n$ points in the interior of $D$. Elements are isotopy classes of homeomorphisms of $D$ that fix the boundary pointwise and leave the set $P_{n}$ invariant. Hence an $n$-braid can be represented by a homeomorphism of $D$. The braid entropy of $\alpha \in B_{n}$ is the infimum of the topological entropy $h(\phi)$, taken over all homeomorphisms $\phi$ representing $\alpha$.

Let $\rho[B(\alpha)(t)]$ denote the spectral radius of the usual Burau matrix of $\alpha$. After a short preparation we will prove the following proposition.

Proposition 4.4. If $\alpha$ is an $n$-braid then for, any positive integer $r$, the algebraic entropy $\gamma_{\alpha, r}$ does not exceed $\sup _{t \in \mathbf{T}^{1}} \rho[B(\alpha)(t)]$.

In $[\mathbf{K o}]$ B. Kolev proved that the entropy $h_{\alpha}$ of any $n$-braid $\alpha$ is bounded below by $\sup _{t \in \mathbf{T}^{1}} \rho[B(\alpha)(t)]$. (As Kolev makes clear, this result is implicit in work of D. Fried $[\mathbf{F r}]$.) The next result follows immediately.

Corollary 4.5. If $\alpha$ is an $n$-braid then, for any positive integer $r$,

$$
\gamma_{\alpha, r} \leq h_{\alpha}
$$

Proposition 4.4 will follow almost immediately from Lemma 4.6, a general result for any $n$-string link that provides insight about the of the associated group $K$.

Lemma 4.6. The $\Lambda$-group $K$ associated to any $n$-string link has a finite presentation such that every element of $\Lambda$ that occurs is a polynomial in $u v$.

Proof. Consider a diagram $D$ for $\alpha$, labeled as in Section 3. We apply Seifert's algorithm (see [Rol], for example), smoothing the crossings to produce pairwise disjoint components: $n$ arcs and some number of circles. Number the components from zero, beginning with the rightmost. Then replace the generators $a, b, \ldots$ of $K$ with $\hat{a}, \hat{b}, \ldots$ such that if a generator $c$ lies on the $k$ th component, then $\hat{c}=c^{v^{k}}$. It is easy to see that the resulting presentation for $K$ has the desired property. 
Example 4.7. We demonstrate the procedure used in the proof of Lemma 4.6 for the 2-string link of Figure 3. The arcs and circles that result from Seifert's algorithm appear in Figure 8. The associated $\Lambda$-group $K$ now has presentation

$$
\begin{aligned}
K=\langle\hat{a}, \hat{b}, \hat{c}, \hat{d}, \hat{e}, \hat{f}, \hat{x}, \hat{y}| \hat{c}^{u v} \hat{a}= & \hat{d}^{u v} \hat{e}, \hat{a}=\hat{d}, \\
& \left.\hat{e}^{u v} \hat{b}=\hat{f}^{u v} \hat{y}, \hat{y}=\hat{e}, \hat{d}^{u v} \hat{f}=\hat{c}^{u v} \hat{x}, \hat{f}=\hat{c}\right\rangle .
\end{aligned}
$$

This should be compared with the earlier presentation (2.2).

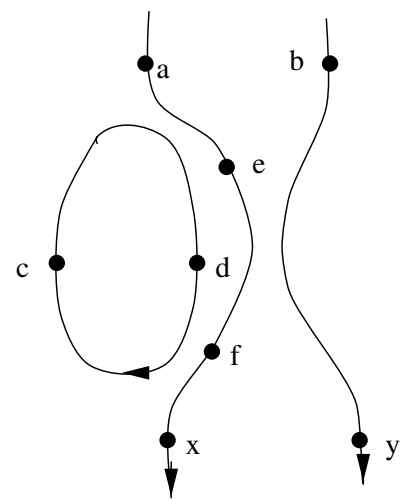

Figure 8. Smoothed Diagram for 2-String Link.

Proof of Proposition 4.4. By Lemma 4.6 we can find a presentation for $K_{a b}$ such that every element of $\Lambda$ that occurs is a polynomial in $u v$. Since $\alpha$ is a braid, each generator of $K_{a b}$ can be expressed as a linear combination of the coordinates $a, b, \ldots$ of $\mathcal{S}$. In particular, each coordinate $x, y, \ldots$ of $\mathcal{T}$ can be written as a linear combination $x^{\prime}, y^{\prime}, \ldots$ with coefficients in $\mathbf{Z}\left[(u v)^{ \pm 1}\right]$. Since $\Delta(s, u, v)$ is the characteristic polynomial of the change of basis matrix, after multiplying by a suitable unit in $\Lambda[s]$ it has the form $\Delta_{n}(u v) s^{n}+\ldots+\Delta_{1}(u v) s+\Delta_{0}(u v)$. Varying $u, v$ over $r$ th roots of unity produces the same set of polynomials that we would get from $\Delta\left(s, 1, t^{-1}\right)$ by varying $t$ over the $r$ th roots of unity. Since $\Delta\left(s, 1, t^{-1}\right)$ is $\operatorname{Det}[s I-B(\alpha)(t)]$, the result follows.

Example 4.8. We return to the 2-string link $\alpha$ in Figure 3. Using the presentation (2.5) we obtain

$$
\begin{aligned}
& L=\langle a, b|(s u v-2 u v+1) a+s\left(u v^{2}-v\right) b=0, \\
& \left.\quad(u v-1) a+\left(s u v^{2}-2 s v+v\right) b=0\right\rangle .
\end{aligned}
$$


A routine calculation shows that

$$
\Delta(s, u, v)=\frac{1}{v}(s-1)\left[\left(2 u v-u^{2} v^{2}\right) s+\left(1-2 u v-2 u^{2} v^{2}\right)\right] .
$$

We have $\Delta(s, 1,1)=(s-1)^{2}, \Delta(s, 1,-1)=(s-1)(3 s-1)$ and $\Delta(s,-1,1)=$ $(s-1)(1-3 s)$. Hence $\gamma_{\alpha, 2}=\log 3$.

We apply algebraic entropy to obtain information about the algebraic topology of branched covers. Let $\alpha$ be a string link with arcs coherently oriented, and $l_{m}$ denote the oriented knot or link obtained as the closure of the product $\alpha^{m}, m \geq 1$. We denote the $r$-fold cyclic cover of $S^{3}$ branched over $l_{m}$ by $M_{r}\left(l_{m}\right)$. The torsion subgroup of $H_{1}\left(M_{r}\left(l_{m}\right) ; \mathbf{Z}\right)$ will be written as $T H_{1}\left(M_{r}\left(l_{m}\right) ; \mathbf{Z}\right)$. The following result extends Theorem 3.2 of $[\mathbf{S i W i}$.

Theorem 4.9. Let $\alpha$ be a string link, and $l_{m}$ the closure of $\alpha^{m}$. Then for any positive integer $r$, the limit

$$
\lim _{m \rightarrow \infty} \frac{1}{m} \log \left|T H_{1}\left(M_{r}\left(l_{m}\right) ; \mathbf{Z}\right)\right|
$$

exists and it is bounded below by the algebraic entropy $\gamma_{\alpha, r}$.

Proof. Consider the $\Lambda[s]$-module $L$ defined at the beginning of the section. Replace each generator $a, b, \ldots$ by an $(r-1)$-tuple $a=\left(a_{0}, \ldots, a_{r-2}\right), b=$ $\left(b_{0}, \ldots, b_{r-2}\right), \ldots$ of generators. Replace $u$ by the operator

$$
S_{r}=\left(\begin{array}{ccccc}
0 & 0 & \ldots & 0 & -1 \\
1 & 0 & \ldots & 0 & -1 \\
0 & 1 & \ldots & 0 & -1 \\
\vdots & \vdots & \ddots & \vdots & \vdots \\
0 & 0 & \ldots & 1 & -1
\end{array}\right),
$$

and replace $v$ by the identity $(r-1) \times(r-1)$-matrix. With these changes, $L$ becomes a $\mathbf{Z}[s]$-module $L_{r}$. From the square relation matrix for $L$ we obtain a square relation matrix $R_{r}$ for $L_{r}$.

We consider the dual group $L_{r}^{\wedge}=\operatorname{Hom}\left(L_{r}, \mathbf{T}^{1}\right)$, a compact abelian group. The action of $s$ on $L_{r}$ induces an automorphism $f_{r}$ on $L_{r}^{\wedge}$. Let $\operatorname{Fix}\left(f_{r}^{m}\right)$ denote the subspace of period $m$ points, and let $\operatorname{Fix}\left(f_{r}^{m}\right)^{\circ}$ be the component of the identity. The arguments of $[\mathbf{S i W i}$ ] can be used to show that the quotient group $\operatorname{Fix}\left(f_{r}^{m}\right) / \operatorname{Fix}\left(f_{r}^{m}\right)^{\circ}$ is isomorphic to $T H_{1}\left(M_{r}\left(l_{m}\right) ; \mathbf{Z}\right.$ ) (see the proof of Theorem 2.10).

By a result of $[\mathbf{L i S c W a}]$ (see Theorem 21.1 of $[\mathbf{S c}]$ ) the limit

$$
\lim _{m \rightarrow \infty} \frac{1}{m} \log \left|\operatorname{Fix}\left(f_{r}^{m}\right) / \operatorname{Fix}\left(f_{r}^{m}\right)^{\circ}\right|
$$

exists and it is equal to the topological entropy $h\left(f_{r}\right)$. Furthermore, Example 18.7 of $[\mathbf{S c}]$ shows that the entropy is equal to $\log \mathcal{M}\left(\operatorname{det} R_{r}\right)$. A calculation 
shows that

$$
\log \mathcal{M}\left(\operatorname{det} R_{r}\right)=\log \prod_{i=1}^{r-1} \mathcal{M}\left(\Delta\left(s, \zeta^{i}, 1\right)\right)
$$

(See Theorem 3.2 of [SiWi] and its proof.) By Lemma 4.6 the right-hand side of (4.2) is equal to the algebraic entropy $\gamma_{\alpha, r}$.

It is natural to ask whether the idea of braid entropy can be generalized for arbitrary string links. If so, then we might anticipate that the quantities $\gamma_{\alpha, r}$ would be lower bounds. We therefore conclude with a question.

Question 4.10. Is there a natural notion of entropy $h_{\alpha}$ for an $n$-string link $\alpha$ ? Can $h_{\alpha}$ be defined as the topological entropy of some homeomorphism?

\section{References}

[AdKoMc] R.L. Adler, A.G. Konheim and M.H. McAndrew, Topological entropy, Trans. Amer. Math. Soc., 114 (1965), 309-319.

[Bi] J.S. Birman, Braids, Links, and Mapping Class Groups, Annals of Math. Studies, No. 82, Princeton University, Princeton, NJ, 1975.

[CaSa] J. Scott Carter and M. Saito, Diagrammatic invariants of knotted curves and surfaces, preprint.

[Fr] D. Fried, Entropy and twisted cohomology, Topology, 25 (1986), 455-470.

[Ja] N. Jacobson, Basic Algebra I and II, W.H. Freeman and Co., New York, 1989.

[Jo] V. Jones, Hecke algebra representations of braid groups and link polynomials, Ann. Math., 126 (1987), 335-388.

[Ka] L.H. Kauffman, Knots and Physics, World Scientific, Singapore, 1991.

[KiLiWa] P. Kirk, C. Livingston and Z. Wang, The Gassner representation for string links, preprint.

[Ko] B. Kolev, Entropie topologique et représentation de Burau, C. R. Acad. Sci. Paris, 309 (1989), 835-838.

[LiTiWa] X. S. Lin, F. Tian and Z. Wang, Burau representations and random walks on string links, Pacific J. Math., 182 (1998) 289-302.

[LiScWa] D. Lind, K. Schmidt and T. Ward, Mahler measure and entropy for commuting automorphisms of compact groups, Invent. Math., 101 (1990), 593-629.

[Ma] K. Mahler, An application of Jensen's formula to polonomials, Mathematika, 7 (196), 98-100.

[Rob] D. Robinson, A Course in Group Theory, Springer-Verlag, Berlin, 1996.

[Rol] D. Rolfsen, Knots and Links, Publish or Perish, Inc., Berkeley, CA, 1976.

[Sc] K. Schmidt, Dynamical Systems of Algebraic Origin, Birkhäuser Verlag, Basel, 1995. 
[SiWi] D.S. Silver and S.G. Williams, Coloring link diagrams with a continuous palette, Topology, 39 (2000), 1225-1237.

Received January 3, 1999 and revised February 28, 1999. Both authors were partially supported by National Science Foundation Grant DMS-9704399.

Department of Mathematics and Statistics

University of South Alabama

Mobile, AL 36688

E-mail address: silver@mathstat.usouthal.edu

University of South Alabama

Mobile, AL 36688

E-mail address: williams@mathstat.usouthal.edu 